\title{
A Rare Case of Traumatic Bilateral Sixth Cranial Nerve Palsy
}

\author{
Hamidon $\mathrm{BB}^{\mathrm{a}}$, Md Shariful $\mathrm{HS}^{\mathrm{a}}$, Nasaruddin $M \mathrm{Z}^{\mathrm{b}}$ \\ aFaculty of Medicine and Health Sciences, Universiti Putra Malaysia \\ ${ }^{b}$ Department of Medicine, Hospital Serdang, Selangor, Malaysia
}

\begin{abstract}
Traumatic bilateral sixth cranial nerve palsy is a rare condition which is typically associated with additional intracranial, skull, and cervical spine injuries. We describe a case of complete bilateral sixth nerve palsy in a 28-year-old female patient after an alleged motor vehicle accident. She had altered level of consciousness but no intracranial lesion or associated skull or cervical spine fracture was detected. In this case, we discussed the differential diagnoses, initial workup, and possible treatment options in cases of traumatic $6^{\text {th }}$ nerve palsy.
\end{abstract}

KEYWORDS: Trauma, nervous system, abducens nerve palsy.

\section{INTRODUCTION}

The term sixth nerve palsy, abducens nerve palsy and lateral rectus palsy are essentially interchangeable. The differential diagnoses for traumatic lateral gaze palsy include brain stem lesions, peripheral nerve injury with or without basilar skull fracture, and lateral rectus muscle injury or entrapment. Physical examination and appropriate imaging tests can differentiate central nervous system, peripheral nerve, and muscular injuries. Isolated loss of lateral gaze with no other cranial nerve signs, no pyramidal tract signs, and no signs of muscular entrapment is thought to result from an injury to the peripheral nerve along its course from the brain stem to the lateral rectus. Any displacement of the brain can cause stretching of the nerve and lead to its palsy. Patients with bilateral sixth nerve palsies complain about binocular horizontal diplopia being worse in the gaze direction of the paretic lateral rectus muscle.

\section{CASE REPORT}

A 28-year-old Malay lady was involved in a motor vehicle accident and suffered head trauma and laceration injuries. She was a pillion rider on a motorcycle. On admission, she was assessed to have a score of 10/15 in the Glasgow coma scale. She also had minor laceration on her face. There were no fractures on further assessment. An urgent brain computerized

Corresponding Author:

Prof. Dr. Hamidon Basri

Deputy Dean (Academic)

Faculty of Medicine and Health Sciences UPM

43400 UPM Serdang

Email: hamidonbasri@gmail.com

Tel: $+603-89472607 / 8$

Fax: $+603-89472585$ tomography (CT) was done. There were no intracranial or extracranial haemorrhage, no mass effect and no oedema. The orbits, sinuses, skull base and calvarium were normal. She regained consciousness about two hours later, when she complained of headache and diplopia in the lateral gaze. Eye examination revealed bilateral complete sixth cranial nerve palsy. The other cranial nerves were intact and fundoscopy examination was normal. Pupillary examination and vision tests were normal. The rest of the neurological examination was normal.

She did not complain of forgetfulness and scored full marks in the mini mental state examination (MMSE) test. Magnetic resonance imaging (MRI) of the brain (including dural sinus assessments by magnetic resonance venography) and orbit was done; which also showed normal results. The patient consented for a lumbar puncture, and cerebrospinal fluid (CSF) analysis was done. The opening pressure was normal at $16 \mathrm{~cm}$ of $\mathrm{H}_{2} \mathrm{O}$. The CSF proteins, cells and biochemical analysis were within normal limits. There was no xanthochromia.

She was treated symptomatically. Recovery from the head trauma was unremarkable; however, the bilateral sixth nerve palsy persisted, and she was discharged from the hospital one week later. At one and three months follow up, her 6th nerve palsy was persistent and complete (Figures 1, 2 and 3). 


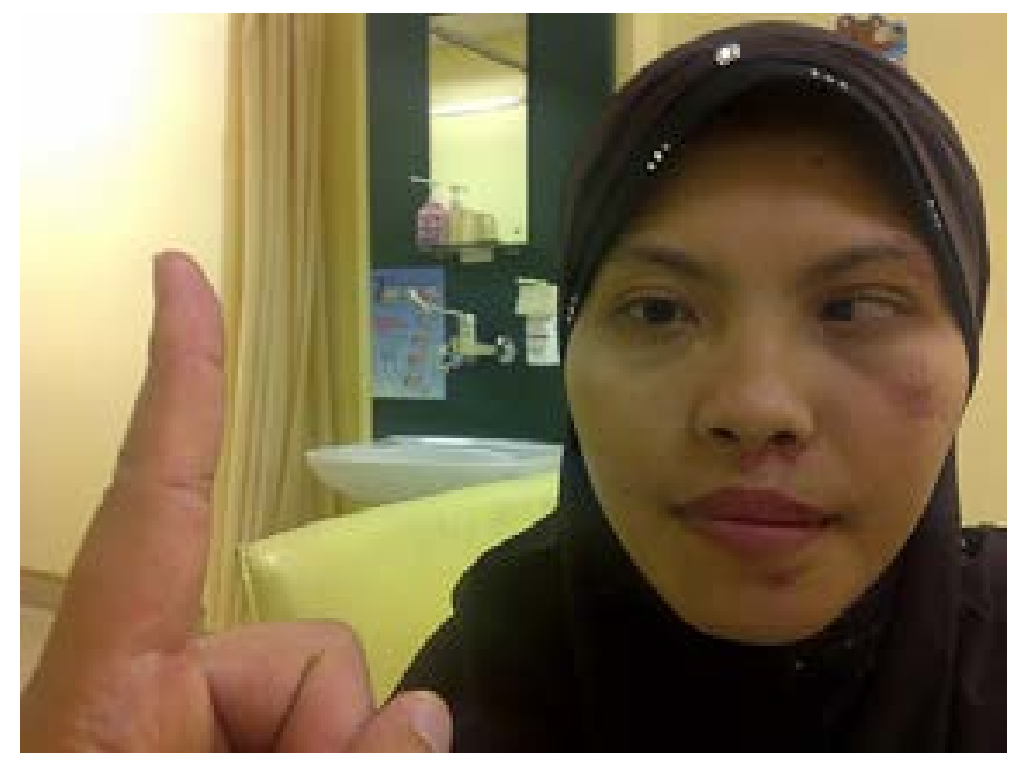

Figure 1: On looking right, there was complete right lateral rectus palsy

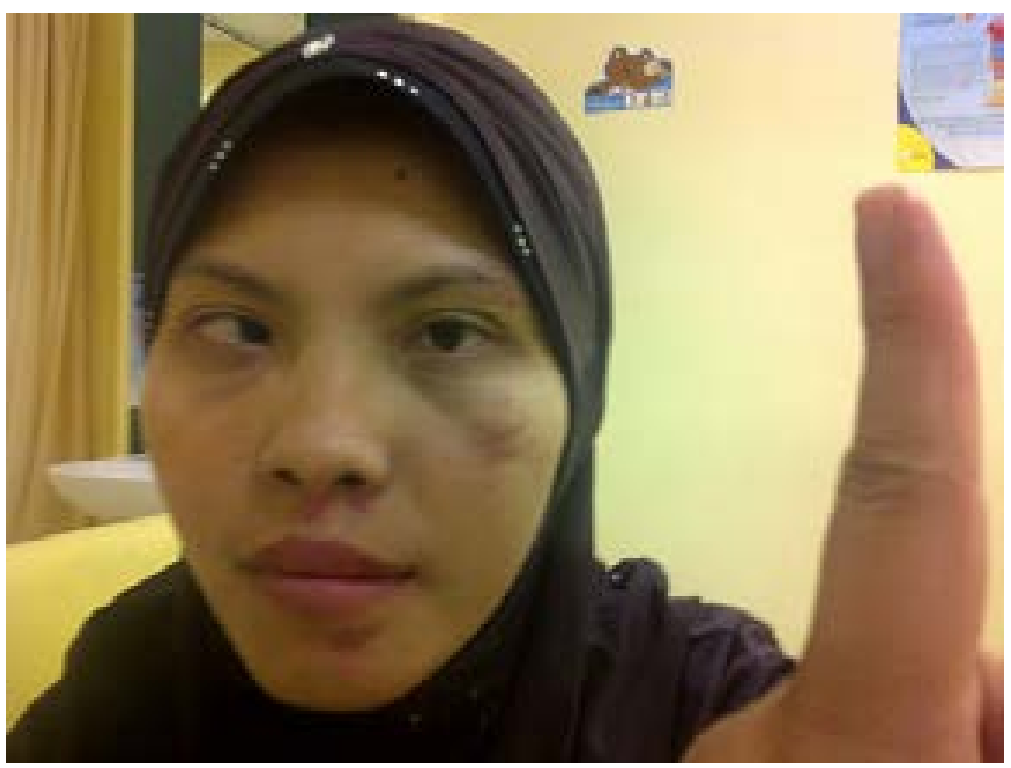

Figure 2: On looking left, there was complete left lateral rectus palsy

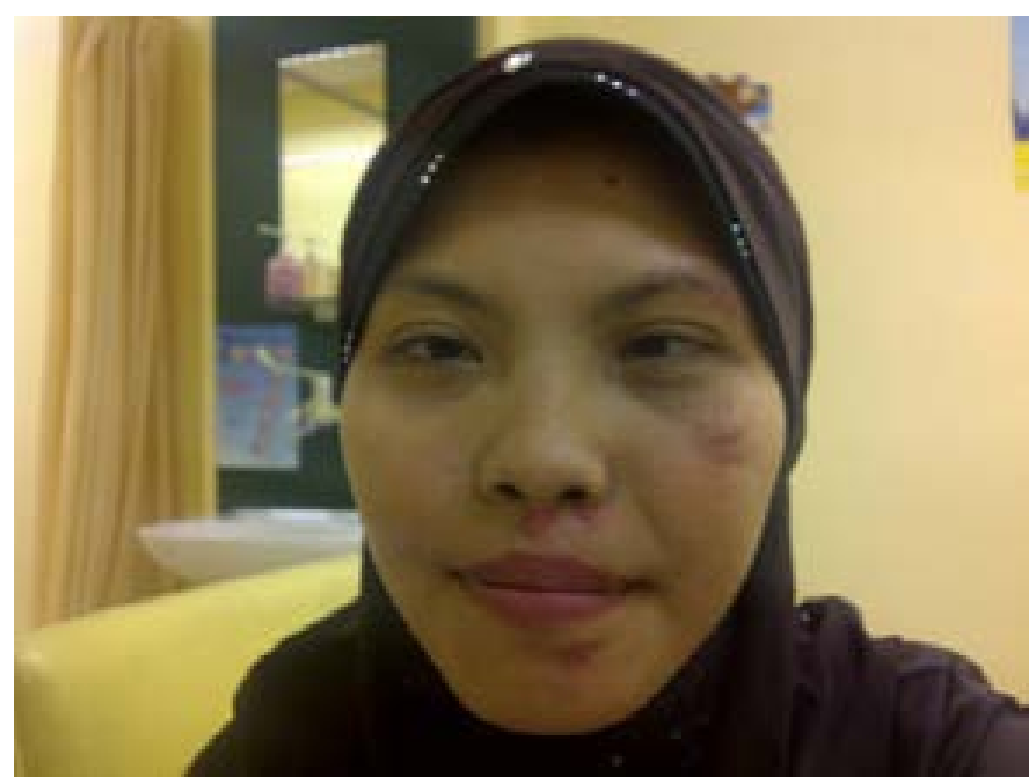

Figure 3: On primary gaze, there was mild bilateral convergent squint 


\section{DISCUSSION}

The sixth cranial nerve (abducens nerve) innervates the lateral rectus (LR) muscle in the ipsilateral eye. Its nucleus is located in the pons, just ventral to the floor of the fourth ventricle and lateral to the medial longitudinal fasciculus (MLF). It has a long intracranial course before it innervates the LR muscle which abducts the ipsilateral eye. Due to its long intracranial course, it is prone to injury. Eye motor paralyses are usually due to direct damage of the sixth cranial nerve, encephalon nuclei or less frequently, diffuse axonal damage. Patients with diffuse axonal injuries usually present with poor conscious level, multifocal neurological damage and carry a poor prognosis.

Sixth cranial nerve palsy may be a false localizing sign and is frequently seen in raised intracranial pressure of any cause. Elevated intracranial pressure can result in downward displacement of the brainstem, causing the sixth cranial nerve to be stretched due to its anatomic location in the Dorello canal. It can also cause pressure or traction of the abducens nerve at the skull base due to its long intracranial course. Therefore, as in this case, it is important to investigate the cause of raised ICP by doing brain imaging (MRI/ $\mathrm{CT}$ ). In cases with bilateral sixth cranial nerve palsy post head trauma, the clinician should consider intracranial haemorrhage, brainstem lesion, basilar skull fracture, peripheral nerve injury, and orbital wall fracture. MRI is superior to the $C T$ in detecting small brain contusions and in imaging of the posterior fossa. However, fractures and overt bleeding are best seen on the CT.

Traumatic bilateral sixth nerve palsy is a rare condition and constitutes only less than $10 \%$ of all cases. ${ }^{1}$ The diagnosis can be considered if brain imaging and CSF examination showed normal results. Usually, there is a significant head trauma with accompanying concussion. ${ }^{2}$ Clinicians must actively look for other injuries in other parts of the body, especially cervical spine fracture. Fortunately, in this case, there was none. In one study, spontaneous recovery for traumatic sixth nerve palsy was $73 \%$ at six months and median time to recovery was 90 days. ${ }^{3}$ Predictors of non-recovery were complete palsy and bilateral palsy. ${ }^{4}$ At three-month follow-up, our patient still had complete sixth nerve palsy. She no longer complained of diplopia and was asymptomatic. Therefore, treatment was only conservative. Treatment options include botulinum toxin injection in both medial rectae to alleviate symptoms, although a definitive solution usually requires surgical intervention.

\section{CONCLUSION}

Bilateral abducens nerve palsy is rare but can occur even in a patient with closed head trauma, without any fractures. It is important to assess intracranial and extracranial injuries. Brain MRI imaging and CSF examinations are the two most important investigations to be done. In cases with complete palsy, the prognosis is usually poor.

\section{REFERENCES}

1. Ruiz-de-Río N, Arbizu-Duralde A, MirandaLloret $P$, Asencio-Durán M, Peralta-Calvo J. Bilateral sixth nerve and left third nerve palsy after head trauma. Arch Soc Esp Oftalmol 2006;81:41-4.

2. Janssen K, Wojciechowski M, Poot S, De Keyser K, Ceulemans B. Isolated abducens nerve palsy after closed head trauma: a pediatric case report. Pediatr Emerg Care 2008;24:621-3.

3. Holmes JM, Droste PJ, Beck RW. The natural history of acute traumatic sixth nerve palsy or paresis. J AAPOS 1998;2:265-8.

4. Holmes JM, Beck RW, Kip KE, et al. Predictors of nonrecovery in acute traumatic sixth nerve palsy and paresis. Ophthalmology 2001;108:1457-60. 